\title{
Correlation of Lipid Peroxidation and Glutathione Levels with Severity of Systemic Lupus Erythematosus: A Pilot Study from Single Center
}

\author{
Tewthanom $K^{1}$, Janwityanuchit $\mathrm{S}^{2}$, Totemchockchyakarn $\mathrm{K}^{2}$, Panomvana $\mathrm{D}^{1}$ \\ ${ }^{1}$ Department of Clinical Pharmacy, Faculty of Pharmaceutical Sciences, Chulalongkorn University, Bangkok, \\ Thailand; ${ }^{2}$ Division of Allergy, Immunology and Rheumatology, Department of Medicine, Faculty of Medicine, \\ Ramathibodi Hospital, Mahidol University, Bangkok, Thailand
}

Received, December 11, 2007; Revised, May 29, 2008; Accepted, June 9, 2008; Published, June 18, 2008.

\begin{abstract}
Pupose. Systemic Lupus Erythematosus (SLE) is a multifactoral chronic autoimmune disease with unidentified etiology. Imbalance of oxidative status is one possible cause of active disease. Plasma malondialdehyde (MDA) and plasma glutathione (GSH) level have been used as a determinate of oxidative status. Limited data has examined these 2 parameters by severity of SLE. Methods. We determined whether there was an association between plasma MDA and plasma GSH level with the severity of SLE. Forty four SLE patients (2 Men and 42 Women) and twenty healthy volunteers (3 Men, 17 women) participated in this study. SLE participants were classified by the severity of disease (mild, moderate or severe). The plasma MDA and plasma Glutathione levels were measured. The correlation of plasma MDA and plasma GSH levels with the severity of SLE disease were determined. Results. Plasma MDA levels with different severity of SLE (mild, moderate, and severe of SLE patients) were not significantly different from those of the control group $(\mathrm{p}=1.0)$. Plasma GSH levels were significantly lower in the moderate and severe SLE groups than the control group $(\mathrm{p}=0.001)$. In addition, a significant correlation between plasma GSH and severity of SLE was observed. (Pearson correlation coefficient $=-0.428, \mathrm{p}$ $<0.001)$. The relationship could be described by the equation GSH level $(\mu \mathrm{M})=(-7.624)$ SLEDAI score + 545.90. Conclusion. A significant correlation between plasma GSH and SLE severity exists that may aid evaluation of the disease severity and usefulness of the treatment of SLE.
\end{abstract}

Corresponding Author: Ph.D. Duangchit Panomvana, Department of Clinical Pharmacy, Faculty of Pharmaceutical Sciences, Chulalongkorn University, Phyathai Road, Bangkok, Thailand, E-mail: duangchit.p@chula.ac.th

\section{INTRODUCTION}

Systemic Lupus Erythematosus (SLE) is a complex chronic immunological disorders with unclear etiologies. There has been attempted to investigate the exactly cause of this disease and many possible theories have been proposed. One of the possible theories about pathology of this disease is an imbalance of the oxidative status of SLE patients. Many researches in animals and in human showed the increasing of lipid peroxidation and decreasing of Glutathione level in animals and patients with this disease [1-10]. However, no research has concentrated on the correlation of lipid peroxidation and Glutathione (GSH) level with different severity of this disease has been presented. Therefore, the aim of this study was to investigate the correlation between lipid peroxidation and the circulating concentrations of GSH and malondialdehyde (MDA), a lipid peroxidation product, with the degree of severity of SLE which may further implied for better understanding of the pathology of this disease.

\section{METHODS}

\section{Patients}

This study was performed at the Division of Allergy, Immunology and Rheumatology of Ramathibody Hospital, Bangkok. All active SLE patients who came to the division during March 2006 to July 2006 and agreed to be included into the project were recruited and classified into 3 groups by severity based on the Systemic Lupus Erythematosus Disease Activity Index (SLEDAI) $[11,12]$ : Mild, score <10; Moderate, score 10-20; Severe, score $>20$.

The classification of severity was made by physicians. Some patients in Moderate and Severe SLE groups who agreed to participate in 
the project were recruited from inpatient department (IPD).

Twenty healthy volunteers with no underlining diseases were also recruited to be a control group of the study.

The project had been approved by the Ethic Committee of the hospital and written informed consents were obtained from all participants.

\section{Blood samples collection and biochemical measurement}

Five $\mathrm{ml}$ of blood samples were drawn and immediately chilled. Plasma was separated by centrifugation at $2^{\circ} \mathrm{C}$ at $3000 \mathrm{rpm}$ for $10 \mathrm{~min}$. The specimens were kept at $-20^{\circ} \mathrm{C}$ and analyzed within 2 weeks.

GSH analysis was performed using a commercially available assay kit (Bioassay Inc, CA, USA). MDA was measured according to the method of Yagi [13], using microplate reader (Victor2 Perkin Elmer Inc,Turku, Finland) as a detector.

\section{Statistical Analysis}

SPSS version 11.5 software (SPSS Inc, Chicago, IL) was used to perform analysis of variance (ANOVA) followed by Bonferoni's test as the post hoc test to identify which mean was different from others, correlation analysis. The Pearson correlation was performed to determine the association of MDA, Glutathione level and the severity of SLE patients. The statistical significant difference was considered when pvalue was less than 0.05 .

\section{RESULTS}

In 5 months, 44 SLE patients (2 men and 42 women) were recruited into the study and were classified into the preset three groups; Mild (20 women), Moderate (15 women), and Severe (2 men and 7 women) (Table 1). The age, smoking habits and SLE duration were not significant difference between groups including Control group (3 Men, 17 women). There was no significant difference in prednisolone and antimalarial drugs use between the patients group. The use of other immunosuppressives, however, was lower in Mild as compared with other two groups. In addition, prednisolone dose (mg/week) was larger (mean \pm SEM) in more severe SLE cases $($ mild $=86.9 \pm 110.0$, moderate $=145.83 \pm$
79.8, severe $=337.5 \pm 187.8$ ). There was significant difference in chloroquine doses between Mild and Moderate groups and also between Moderate and Severe groups. Similarly, the dose of cyclophosphamide was significantly higher in the moderate cases as compared with others.

All of the control volunteers were healthy as determined by physicians having normal diet and agility with no underlining diseases such as cardiovascular or allergic disorders that may affect the oxidative status.

\section{GSH and MDA concentrations in Plasma}

GSH. As depicted in Figure 1 and Table 2, there were significant difference in the mean and $95 \%$ confidence interval (CI) value of plasma GSH concentrations of patients with different severity of SLE. The values were significantly higher in Moderate and Severe as compared with Control. There was no significant difference between Mild and Control.

MDA. The mean and 95\% CI were not significantly different among the four groups (Figure 2, Table 2).

\section{Correlation of Plasma MDA and GSH with Severity of SLE}

There was a significant negative correlation between plasma GSH and severity of SLE (Pearson correlation coefficient $=-0.479$, $\mathrm{p}=0.001$ ). The regression equation to predict the GSH level based on the severity of SLE disease was, GSH level $(\mu \mathrm{M})=-132.25$ Group severity + $655.578 \quad(p=0.001)$. Where dummy or indicator variables had been used for group severity 1 for Mild, 2 for Moderate and , 3 for Severe. These numbers do not represent any actual measurements; they simply identify the categories of the nominal random variable.

There was a positive but not significant trend for correlation between plasma MDA concentration and the SLE severity (Pearson correlation coefficient $=0.166, \mathrm{p}=0.281$ ) .

In addition, the correlation between scores oxidative status parameters and SLE severity were re-analyzed using the continuous SLEDAI scores in place of the categorized group severity scale. Nearly the same results were observed. 
Significant correlation between SLEDAI and GHS levels was observed (Pearson correlation coefficient $=-0.428, \mathrm{p}<0.001$ ) with the following equation: GSH level $(\mu \mathrm{M})=-7.624$ SLEDAI +545.90 .
MDA levels were showed a trend for relations with SLEDAI score, however, the correlation did not reach significance (Pearson correlation coefficient $=0.174, \mathrm{p}=0.084$ ).

Table 1. The demographic data of subjects ${ }^{\text {a }}$

\begin{tabular}{|c|c|c|c|c|c|}
\hline & \multirow[b]{2}{*}{$\begin{array}{c}\text { Control } \\
(n=20)\end{array}$} & \multicolumn{3}{|c|}{ SLE patients } & \multirow[b]{2}{*}{ p-value } \\
\hline & & $\begin{array}{c}\text { Mild } \\
(\mathrm{n}=20)\end{array}$ & $\begin{array}{l}\text { Moderate } \\
(\mathrm{n}=15)\end{array}$ & $\begin{array}{c}\text { Severe } \\
(\mathrm{n}=9)\end{array}$ & \\
\hline Age, years & $44.9 \pm 15.6$ & $37.7 \pm 13.2$ & $36.3 \pm 11.5$ & $39.8 \pm 13.6$ & 0.573 \\
\hline Smoking & No & No & No & No & 1.00 \\
\hline SLE duration, years & 0 & $4.5 \pm 3.3$ & $5.73 \pm 6.3$ & $6.6 \pm 3.9$ & 1.00 \\
\hline SLEDAI score & 0 & $3.4 \pm 1.8$ & $13.3 \pm 1.3$ & $37.8 \pm 8.7$ & $0.0001 *$ \\
\hline \multicolumn{6}{|l|}{ Drugs being used: } \\
\hline Prednisolone (\%) & 0 & 85 & 100 & 100 & 0.151 \\
\hline Prednisolone dose, mg/wk & 0 & $86.9 \pm 110.0$ & $146 \pm 80$ & $338 \pm 188$ & $0.0001 *$ \\
\hline Antimalarial (\%) & 0 & 65 & 87 & 67 & 0.335 \\
\hline CQ dose , mg/wk & 0 & $400 \pm 676^{b}$ & $1183 \pm 765^{\mathrm{b}, \mathrm{c}}$ & $389 \pm 772^{c}$ & $0.0001 *$ \\
\hline HCQ dose , mg/wk & 0 & $410 \pm 614$ & $160 \pm 429$ & $622 \pm 738$ & $0.007 *$ \\
\hline Other Immunosuppressives (\%) & 0 & 25 & 67 & 56 & 0.042 \\
\hline AZA dose , mg/wk & 0 & $35.0 \pm 107.7$ & $140 \pm 178$ & $117 \pm 175$ & $0.006 *$ \\
\hline CYP dose, mg/wk & 0 & $35.0 \pm 108^{\mathrm{d}}$ & $227 \pm 350^{\mathrm{d}}$ & $77.8 \pm 154$ & $0.007 *$ \\
\hline
\end{tabular}

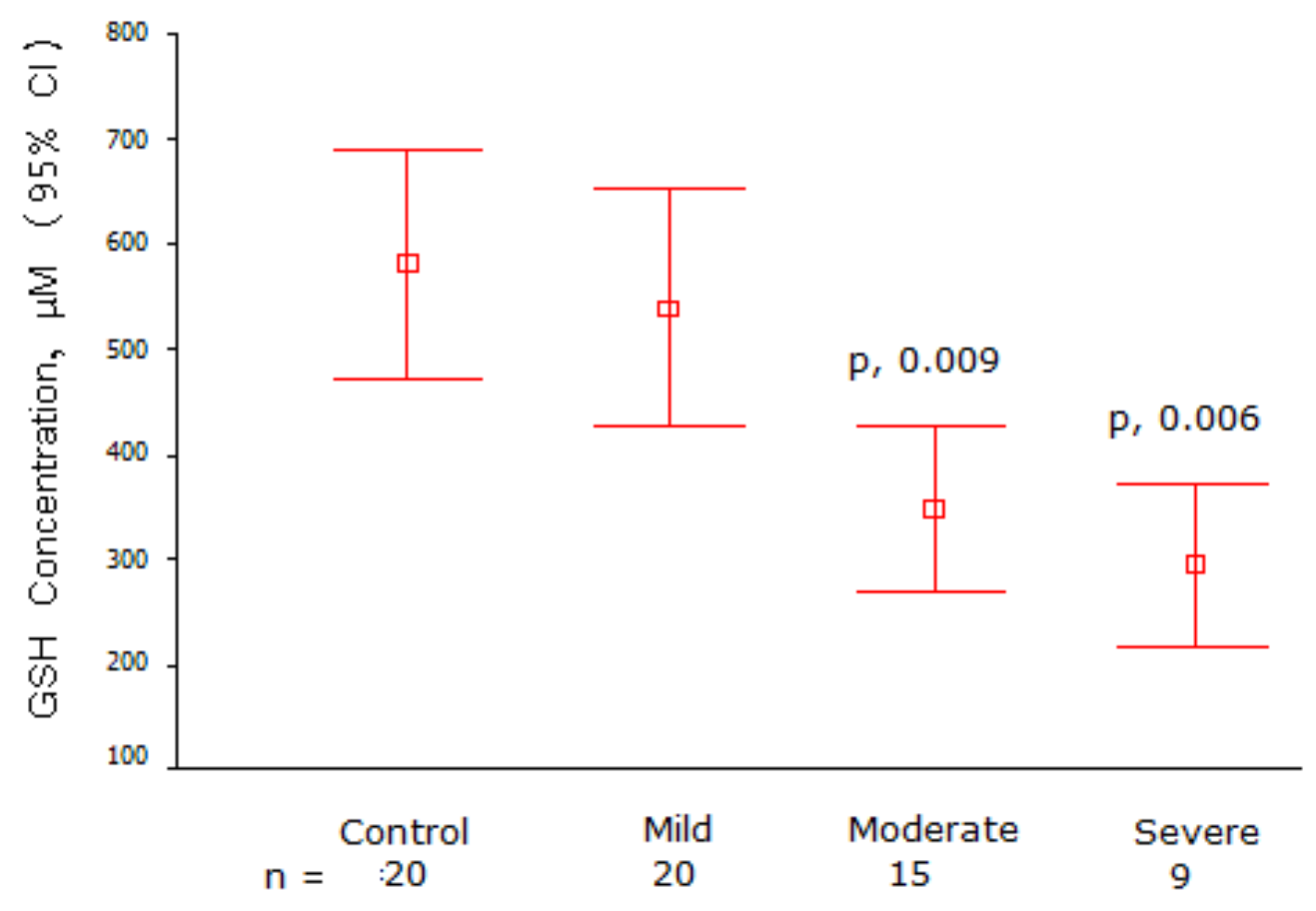

Figure 1. The mean and 95\% CI of plasma GSH concentrations $(\mu \mathrm{M})$ of patients with different severity of SLE $(\mathrm{p}<0.05$, significantly different from Control) 


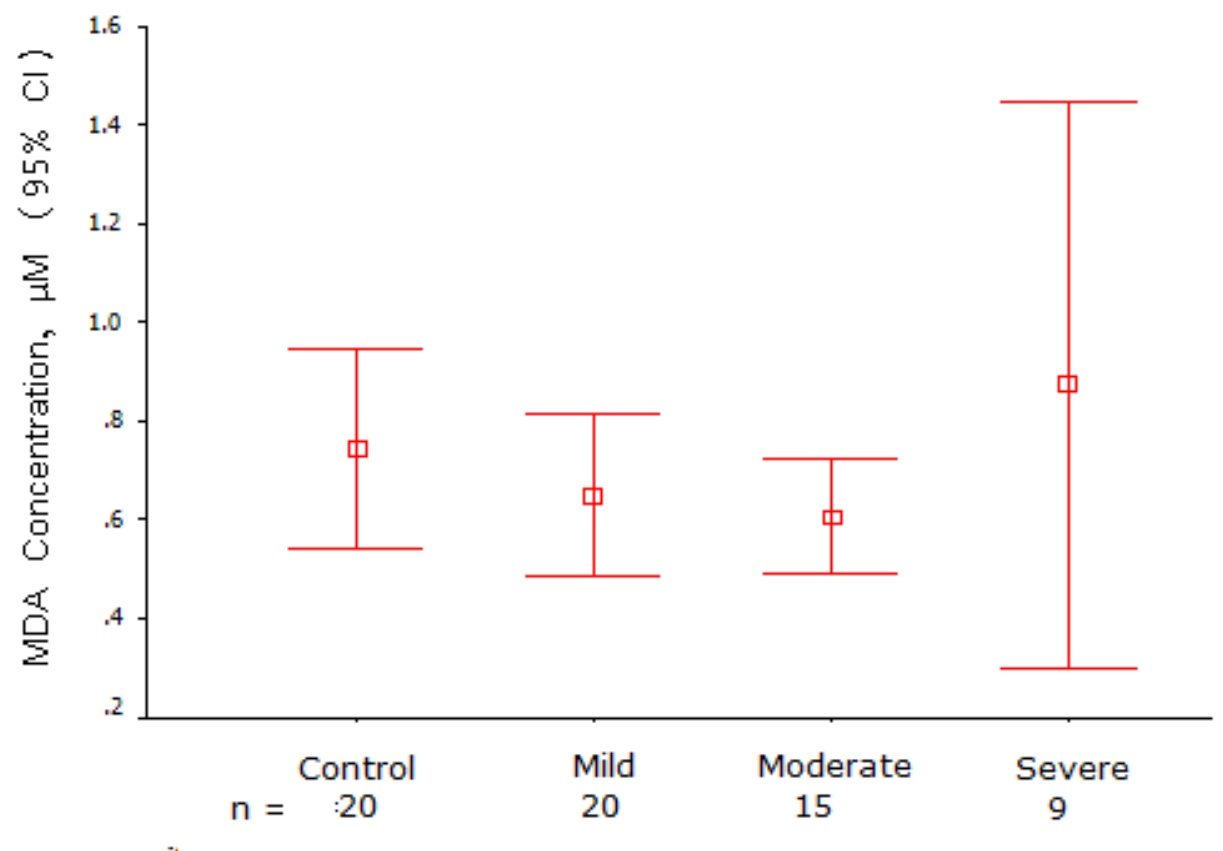

Figure 2. The mean and 95\% CI of Plasma MDA concentration $(\mu \mathrm{M})$ of patients with different severity of SLE.

Table 2. Mean and SEM of oxidative status parameters in SLE and control patients

\begin{tabular}{lcccc}
\hline & $\begin{array}{c}\text { Control } \\
(\mathrm{N}=20)\end{array}$ & $\begin{array}{c}\text { Mild SLE } \\
(\mathrm{N}=20)\end{array}$ & $\begin{array}{c}\text { Group } \\
\text { Moderate SLE } \\
(\mathrm{N}=15)\end{array}$ & $\begin{array}{c}\text { Severe SLE } \\
(\mathrm{N}=9)\end{array}$ \\
\hline Glutathione, $\mu \mathrm{M}$ & $583 \pm 52$ & $540 \pm 54$ & $348 \pm 37$ & $295 \pm 34$ \\
p value against Control & & 0.915 & 0.009 & 0.006 \\
Malondialdehyde, $\mu \mathrm{M}$ & $0.74 \pm 0.097$ & $0.65 \pm 0.078$ & $0.61 \pm 0.055$ & $0.88 \pm 0.249$ \\
$p$ value against Control & & 1.00 & 1.00 & 1.00 \\
\hline
\end{tabular}

\section{DISCUSSION}

Base on the evidence that imbalance of oxidative status is associated with the pathology of SLE [1, $5,10,14,15]$, this study was performed to determine the correlation of oxidative status parameters and the degree of severity of SLE disease. A significant correlation was observed between plasma GSH and SLE severity. However, our work had several limitations. First, the blood sample from every patient was not collected at the same time of the day and this was a single point measurement. Second, there were several other factors which might have influence on GSH levels, for example, duration of SLE, and the use of prednisolone, antimalarial, and other immunosuppressive medications. The duration of SLE, however, may be ruled out as there was no significant difference between groups with this regard (Table 1). The use of prednisolone, antimalarials and other immunosuppressive medications as confounding factors might also be ruled out as, their use is expected to increase and not, as we observed, decrease GSH concentrations.

For MDA levels, the relationship between MDA levels and SLEDAI score was less obvious 
than that reported previously [6]. This may be due to the effect of prednisolone on lipid peroxidation [16] and/or other immunosuppressive medications, since the recruited patients in the previous report were studied before the starting of SLE treatment. That implies that the treatment may result in normalization of MDA levels by improving the oxidative status.

In a recent study of a relationship of oxidative status and SLE severity, a different oxidative stress parameter, i.e., isoprostane has been monitored that is related to the fatigue symptom [17] . Therefore, this study is the first to report the relationship between glutathione level and SLE severity.

In conclusion, a significant correlation between plasma GSH and SLE severity exists that may aid evaluation of the disease severity and usefulness of the treatment of SLE.

\section{ACKNOWLEDGEMENT}

The authors are grateful to Chulalongkorn University for financial support. Special grateful acknowledgement was regarded to all health care members (nurses, residents, medical staffs, officers) at Ramathibodi hospital who provided a kind assistance during the study.

\section{REFERENCES}

[1]. Kurien BT, Scofield RH. Lipid peroxidation in systemic lupus erythematosus. Indian J Exp Biol 2006;44:349-56.

[2]. Frostegard J, Svenungsson E, Wu R, Gunnarsson I, Lundberg IE, Klareskog L, et al. Lipid peroxidation is enhanced in patients with systemic lupus erythematosus and is associated with arterial and renal disease manifestations. Arthritis Rheum 2005;52:192200.

[3]. Hsieh CC, Lin BF. The effects of vitamin E supplementation on autoimmune-prone New Zealand black x New Zealand white F1 mice fed an oxidised oil diet. $\mathrm{Br} \mathrm{J}$ Nutr 2005;93:655-62.

[4]. Auborn KJ, Qi M, Yan XJ, Teichberg S, Chen $\mathrm{D}$, Madaio MP, et al. Lifespan is prolonged in autoimmune-prone (NZB/NZW) F1 mice fed a diet supplemented with indole-3-carbinol. J Nutr 2003;133:3610-3.

[5]. Kurien BT, Scofield RH. Free radical mediated peroxidative damage in systemic lupus erythematosus. Life Sci 2003;73:165566.

[6]. Taysi S, Gul M, Sari RA, Akcay F, Bakan N. Serum oxidant/antioxidant status of patients with systemic lupus erythematosus. Clin Chem Lab Med 2002;40:684-8.

[7]. Ames PR, Alves J, Murat I, Isenberg DA, Nourooz-Zadeh J. Oxidative stress in systemic lupus erythematosus and allied conditions with vascular involvement. Rheumatology (Oxford) 1999;38:529-34.

[8]. Michel P, Eggert W, Albrecht-Nebe H, Grune $\mathrm{T}$. Increased lipid peroxidation in children with autoimmune diseases. Acta Paediatr 1997;86:609-12.

[9]. Mohan IK, Das UN. Oxidant stress, antioxidants and essential fatty acids in systemic lupus erythematosus. Prostaglandins Leukot Essent Fatty Acids 1997;56:193-8.

[10]. Tanaseanu S, Serban MG, Nita V. Lipid peroxidation and the activity of some antioxidant enzymes in patients with systemic vasculitides treated with corticoids. Rom J Intern Med 1994;32:47-50.

[11]. Institueforalgorithmicmedicine. Algorithm for systemic lupus erythematosus (SLE) disease activity index. The institue for algorithm medicine, 2007.

[12]. Griffiths B, Mosca M, Gordon C. Assessment of patients with systemic lupus erythematosus and the use of lupus disease activity indices. Best Pract Res Clin Rheumatol 2005;19:685708.

[13]. Yagi K. Simple assay for the level of total lipid peroxides in serum or plasma. Methods Mol Biol 1998;108:101-6.

[14]. Bae SC. , Kim SJ. , Sung MK. . Impaired antioxidant status and decreased dietary intake of antioxidants in patients with systemic lupus erythematosus. Rheumatol Int 2002;22:238-43.

[15]. Donne DI , Rossi R , Colombo R , Giustarini $\mathrm{D}$, Milzani A Biomarkers of oxidative damage in human disease. Clin Chem 2006;52:601-23.

[16]. Kouno T, Egashira T, Takayama F, Kudo Y, Yamanaka Y. Effect of methylprednisolone on plasma lipid peroxidation induced by lipopolysaccharide. Jpn J Pharmacol 1994;64:163-9.

[17]. Avalos I, Chung CP, Oeser A, Milne GL, Morrow JD, Sadik GT, et al. Oxidative stress in systemic lupus eythematosus:relationship to activity disease activity and symptoms. Lupus 2007;16:195-200. 